\title{
Conductance Measurement of Metal Nano Contacts at Mechanically Controllable Break Junctions in Solution
}

\author{
Nobuo Sekiguchi, Manabu Kiguchi *, and Kei Murakoshi \\ Division of Chemistry, Graduate School of Science, Hokkaido University, Sapporo, 060-0810, Japan
}

Fax: 81-11-706-4811, e-mail: kiguti@sci.hokudai.ac.jp

\begin{abstract}
Electric conductance of the $\mathrm{Au}$ nanowires was investigated in $0.1 \mathrm{M} \mathrm{Na}_{2} \mathrm{SO}_{4}$ solution under electrochemical potential control using a mechanically controllable break junction setup with an electrochemical cell (EC-MCBJ). The conductance histogram exhibited a series of peaks, suggesting the existence of the stable diameter of the nanowires. Two different slopes were obtained in the plot of the stable diameter as a function of the peak index. The two slopes could be explained by the combination of the electronic and atomic shell effects. We could observe the shell structures in the conductance histogram of the Au nanowires under electrochemical potential control for the first time.
\end{abstract}

Key words: metal nanowire, conductance quantization, electrochemical potential, shell effect

\section{INTRODUCTION}

There is growing interest in metal nanowires due to their unique properties and their potential application in microelectronic devices [1]. Electrical conductance through a metal nanowire on an atomic scale is expressed by $G=2 e^{2} / h \sum T_{i}=G_{0} \sum T_{i}$ where $T_{i}$ is the transmission probability of the $i$-th conductance channel, $e$ is the electron charge, $h$ is Plank's constant, and $G_{0}$ is a unit of the quantized conductance. Since conductance quantization depends on the atomic structure of a nanowire, the atomic structures of the metal nanowires could be studied by conductance measurements. The radius $R$ of alkali or noble metal nanowires is related with the conductance $(G)$ using the semi-classical formula (Sharvin formula)

$$
G=G_{0}\left[\left(\frac{k_{F} R}{2}\right)^{2}-\frac{k_{F} R}{2}+\frac{1}{6}\right]
$$

where $k_{F}$ is the Fermi wave vector [1]. Recently, it has been observed that alkali and noble metal nanowires exhibit exceptional stability for the certain diameters by conductance measurements [2-8]. The series of stable diameter were explained by the electronic and atomic shell effects. The electronic shell effect originates from that nanowires become stable when the length of the allowed trajectory of electrons inside a cylindrical cavity (nanowire) agrees with the integer multiple of the Fermi wavelength. With an increase in the diameter, the configuration energy (atomic shell effect) becomes more important than the electronic energy contribution (electronic shell effect). Neglecting the electronic energy contribution, the optimal section of a fcc crystal is a density packed wire with four (111) and two (100) facets. The atomic shell effect originates from the completion of additional atomic layers for the nanowire with this optimal section. Either electronic or atomic shell effect is the dominant factor to determine the stable nanowires. The relative energy difference between the electronic free energy and the surface energy determines the transition point. Transition between two states, in which one of the energies are dominant, have been observed for alkali and noble metal nanowires in ultra high vacuum (UHV) and air [2-8].

Recently, the electrochemical method has been recognized to be a powerful approach to fabricate metal nanowires [9-12]. Electrochemical potential determines the potential energy of the electrons of nanowires, resulting in the control of the bonding strength between the metal atoms and the interaction of the metal at surface with molecules of surrounding medium. These facts make possible to fabricate stable metal nanowires, which has not been prepared in UHV. Fabrication of stable $\mathrm{Au}, \mathrm{Ag}, \mathrm{Cu}, \mathrm{Pb}, \mathrm{Fe}, \mathrm{Co}, \mathrm{Ni}$, and $\mathrm{Pd}$ nanowires and interesting phenomena characteristic of the electrochemical system have been reported in solution [9-12]. It is attractive to study the shell effects in metal nanowires in solution. Since potential energy of electrons in nanowires can be defined by applied electrochemical potential, the appearance of the electronic shell effect could be controlled by adapting appropriate electrochemical potential. The atomic shell effect can be also controlled by the electrochemical potential. The surface stress and structure of $A u(100)$ and (111) electrodes were studied in solution [13]. The surface stress 
decreases as the potential was scanned from -400 $\mathrm{mV}$ vs. SCE to positive, and reconstruction occurs at the potential regime more positive than $700 \mathrm{mV}$. This result indicates that the surface stress can be defined by the electrochemical potential. The surface stress and structure play the decisive role in appearance of the atomic shell effect. Thus, novel atomic shell effect which has not been observed in UHV could be expected under electrochemical potential control. In addition, the transition between two states, in which one of the effects are dominant could be varied with the electrochemical potential. In the present study, we have, thus, mechanically fabricated $\mathrm{Au}$ nanowires in $0.1 \mathrm{M} \mathrm{Na} \mathrm{NO}_{4}$ solution using mechanically controllable break junction setup with an electrochemical cell (electrochemical mechanically controllable break junction system: EC-MCBJ). The shell effect in the $\mathrm{Au}$ nanowires was investigated by conductance measurements under electrochemical potential control.

\section{EXPERIMENTAL}

The measurements have been performed using MCBJ technique [14]. The bending beam was a plate of phosphor bronze about $1 \mathrm{~mm}$ thick, 20 $\mathrm{mm}$ long and $8 \mathrm{~mm}$ wide. The surface of the plate was insulated with a thin polymer foil. The notched Au wire was glued on the bending beam by two drops of epoxy adhesive. The drops and the metal wire were covered with wax except the notched area. The whole assembly was mounted on a three-point bending mechanism consisting of a stacked piezo-element and two fixed counter supports. By bending the substrate, the top surface of the substrate was expanded and the wire was finally broken forming two separated electrodes. By relaxing the bending of the substrate, the contact could be re-established. Figure 1 shows the schematic view and photograph of the present EC-MCBJ setup. The electrochemical cell was made of Diflon. The cell was tightly pressed against the substrate. The electrolyte solution was $0.1 \quad \mathrm{M} \mathrm{Na}_{2} \mathrm{SO}_{4}$. The electrochemical potential $(\phi)$ of the Au wire was controlled with respect to a $\mathrm{Ag} / \mathrm{AgCl}$ reference electrode using a four-electrodes potentiostat (twin potentiostat HECS990C: Fuso Co.). A 0.30 $\mathrm{mm}$ diameter $\mathrm{Pt}$ wire was used as a counter electrode. Conductance was measured during the breaking process under an applied bias of $20 \mathrm{mV}$ between the two working electrodes.

\section{RESULTS AND DISCUSSION}

Figure 2 (a) shows the typical conductance traces of the Au nanowires measured at $\phi=+500$ $\mathrm{mV}$ in $0.1 \mathrm{M} \mathrm{Na}_{2} \mathrm{SO}_{4}$. The stretching length was estimated by considering the geometry of the present MCBJ setup and piezo displacement. The ratio between the relative displacement of the two electrodes and displacement of piezo element was $5 \times 10^{-3} \quad[14]$. The conductance gradually decreased with the stretch length (distance

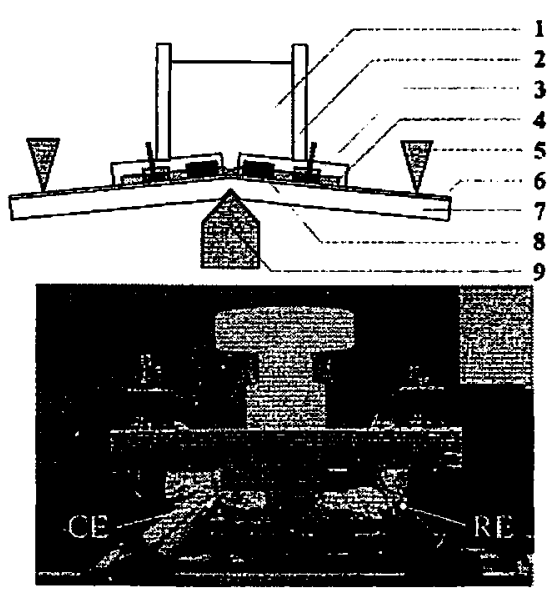

Fig. 1. Schematic side view and photograph of EC-MCBJ for measuring electric conductance of the metal nanowire under electrochemical potential control; CE: counter electrode and RE: reference electrode. (1) electrolyte solution, (2) electrochemical cell, (3) coating wax, (4) notched Au wire, (5) two fixed counter supports, (6) polymer foil, (7) bending beam of phosphor bronze, (8) epoxy adhesive, and (9) piezo element.

between the two electrodes), which could reflect length of $\mathrm{Au}$ nanowire. At low conductance regime, the conductance decreased in a stepwise fashion with each step occurring at an integer multiple of $G_{0}$.

Figure 2(b) shows the conductance histogram obtained from 10000 individual conductance traces. The histogram shows peaks centered at 1.0 (peak1), 2.0 (peak2), 3.0 (peak3), 4.5 (peak4), 7.0 (peak5) and $9.5 G_{0}$ (peak6), together with weak features at higher conductance regime. While the conductance of the first, second, and third peaks were just multiple of $G_{0}$, the conductance of the higher conductance peaks shifted to the lower conductance value. This shift primarily originated from the back scattering of the electrons on defects in the Au nano constrictions [1]. Each peak indicated the formation of a stable atomic configuration. To resolve the features at high conductance regime, the second derivative of the histogram was carried out. The minima of the derivatives corresponded to the peak of the original histogram. Figure 3 shows the peak positions in units $k_{F} R$ as a function of peak index $(n)$ Here, this plot is called as $n-k_{F} R$ plot. $k_{F} R$ was obtained with the help of Eq. (1). In Fig. 3, two different slopes are evident, one slope is a 0.9 for $n<4$ and another slope is 0.5 for $n>4$. Liner increment of $k_{F} R$ to the peak index proves the periodic appearance of the stable nanowires. This result indicates that we could observe successfully the shell effect in the Au nanowires under electrochemical potential control for the first time. The origin of these slopes is discussed in the following. 
First, the slope of 0.9 is discussed using a theoretical mode. The model considers a nanowire as an infinitely long cylinder assuming
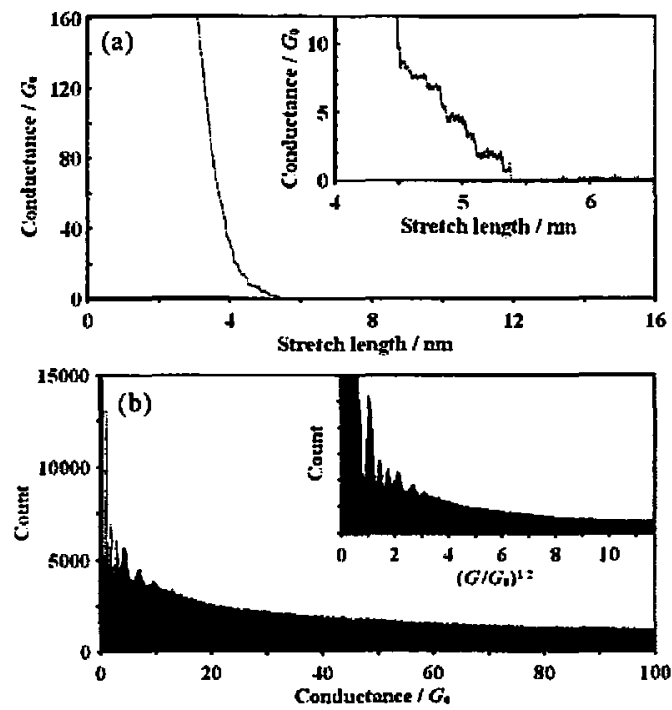

Fig. 2. (a) Typical conductance trace and (b) corresponding conductance histogram of $\mathrm{Au}$ nanowires in $0.1 \mathrm{M} \mathrm{Na}_{2} \mathrm{SO}_{4}$ at $+500 \mathrm{mV}$. Inset shows the histogram plotted as a function of the square root of the conductance.

the average valence electron density of the metal. In this model, a cylinder with a radius $(R)$ is stable when the length of the trajectory $(L)$ of electrons inside the cylinder agrees with the integer multiple of the electron wave length $\left(\lambda_{F}\right)$. This matching condition is represented as

$L / \lambda_{F}=n(2)$

Therefore, the distribution of stable cylinders as a function of $R$ has a periodic structure. The lowest order semi-classical orbits of electrons are diametric, triangular and square orbits, whose length are $4 R, 3 \sqrt{3} R, 4 \sqrt{2} R$, respectively. For the diametric orbit, the matching conditions is $L$ $/ \lambda_{F}=4 R / \lambda_{F}=(2 / \pi)\left(k_{F} R\right) \approx 0.64\left(k_{F} R\right)=n$. Similarly for the triangular orbit, the matching condition is $L / \lambda_{F}=3 \sqrt{3} R / \lambda_{F} \approx 0.83\left(k_{F} R\right)=n$. For the square orbit, the condition is $L / \lambda_{F}=4 \sqrt{2} R / \lambda_{F}$ $\approx 0.90\left(k_{F} R\right)=n$. The $n-k_{F} R$ plot should show slopes of 1.56, 1.20 and 1.11 for diameter, triangular and square orbits, respectively. In the previous studies for the Au nanowires, the slope of 1.1 or 0.9 were observed in the $n-k_{F} R$ plot $[2,3,8]$. The Fourier transform of the conductance histogram showed a peak centered at a frequency of $1 / \Delta k_{F} R=0.92[2,3]$. These values were close to the values expected from the triangular and square orbits, although there were some differences. Therefore, the orbits that have the significant contribution for the Au nanowires are the triangular and square orbits. In the present study, the slope of 0.9 was observed in the $n-k_{F} R$ plot. This value agrees with the value obtained in the previous studies, and thus, the slope of 0.9 observed in the present study would originate from the similar electronic shell effect of the Au nanowire in an electrolyte solution under electrochemical potential control. The deviation from the expected value could be explained by the conductance lowering due to backscattering on defects in or near the nanowire. Sulfate anions

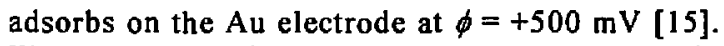
The adsorbed anion onto the Au nanowire would lower the conductance, leading to the small slope.

Second, another slope of 0.5 is discussed by considering the surface energy of the nanowire. Both electronic free energy and surface energy of nanowires oscillate as a function of a diameter of the nanowire. The oscillation amplitude of the electronic free energy is inversely proportional to the diameter while the amplitude of surface energy is roughly constant [2]. The surface energy becomes more important than the free energy at larger diameter, and thus, the stability of the nanowire depends on the surface energy at larger diameter. Since theoretical calculation predicts that the [110] direction is a preferred

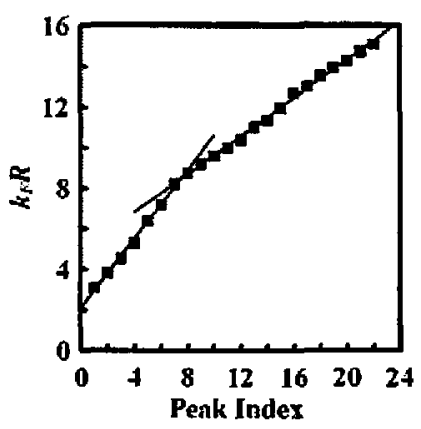

Fig. 3. Position of the peaks in fig. 2 as a function of peak index. The position is converted to $k_{F} R$ with the help of Eq. (1).

orientation for the process of thinning down of a nanowire [16], the nanowire could be considered to be along the [110] axis. The optimal cross section of the wire along [110] is the hexagonal cross section with four (111) and two (100) facets, which has the lowest surface energy. Stable configurations are achieved when the facets are completely covered with atoms. This is the origin of the atomic shell effect. While completing a full atomic shell gives a rather long period of $\Delta k_{F} R=$ 2.85 , completing a single facet gives a six times smaller period of $\Delta k_{F} R=0.48$. The resulting periodicity leads to the observation of the slope of 0.5 or 0.4 in the $n-k_{F} R$ plot as shown in the previous studies for the Au nanowires $[2,3,8]$. In the present study, the slope of 0.5 was observed in the $n-k_{F} R$ plot. This value agrees with the predicted value and one obtained in the previous studies, and thus, the slope of 0.5 would originate from the atomic shell effect.

Oshima et al. reported another series of stable diameters of $\mathrm{Au}$ nanowires using transmission electron microscope (TEM) [17]. They observed 
stable $n-n^{\prime}$ helical multi shell (HMS) $\mathrm{Au}$ nanowires, where $n$ and $n^{\prime}$ are the number of atomic rows of $\mathrm{Au}$ in the outer and inner shells. The conductance values for the HMS wires are 7.6-8.1 $G_{0}$ for the 11-4 HMS wire, 8.4-9.7 $G_{0}$ for the 12-5 HMS wire, 10.2-10.6 $G_{0}$ for the 13-6 HMS wire, and so on. While some of them agreed with our results obtained by MCBJ, our results did not completely agree with their results using TEM [2]. At the present stage, we could not exclude the possibility of the formation of the helical nanowire, but the peak position in the histogram agreed with the theoretically predicted position assuming the shell effects. So, the shell effect would be dominant factor determining the diameter of $\mathrm{Au}$ nanowires in the present study. The difference in the experimental results would be attributed to the different experimental methods for forming the nanowires.

Finally, transition between two states, in which one of the shell effects are dominant, is discussed. In UHV, the transition occur around $10 G_{0}$ and the transition conductance varies between different experiments. In some cases, either atomic or electronic shell effect was observed even for the same metal. [2]. In the present study, the transition conductance was constant around $16 G_{0}$. The larger transition conductance value and its small variation in solution is possibly due to molecular adsorption on the nanowire. As discussed in the previous section, the surface energy plays a decisive role in the appearance of the atomic shell effects. At $\phi=+500 \mathrm{mV}$ in $0.1 \mathrm{M}$ $\mathrm{Na}_{2} \mathrm{SO}_{4}$, sulfate anions would adsorb on the $\mathrm{Au}$ nanowire [15]. The molecular adsorption decreases the surface energy of the nanowire [18], leading to the delay of the transition from the state in which electronic shell effect is dominant to the state in which atomic shell effect is dominant. The variation of the transition conductance is due to the difference of the local crystalline orientation at breaking events [2]. If the certain atomic configuration could be stabilized by molecular adsorption, the transition conductance would be constant. Actually, we have shown that stable mono atomic contacts of $\mathrm{Fe}, \mathrm{Co}, \mathrm{Ni}, \mathrm{Pd}$, or $\mathrm{Au}$ [9-12] could be stabilized under electrochemical potential control, while they can not be prepared in UHV at room temperature. The molecular adsorption could define the certain atomic configuration at even larger diameter, leading to small variation in the transition conductance value. The structure and surface energy of metal electrodes could be controlled by electrochemical potential. The present results suggest that the appearance of the atomic shell effect could be controlled by the electrochemical potential. The detailed investigation on the electrochemical potential dependence is in progress in our laboratory. Since the peak labeling process has remained obscure especially at higher conductance regime, the improving of this process is also in progress.

\section{SUMMARY}

Au nanowires were mechanically fabricated in $0.1 \mathrm{M} \mathrm{Na} \mathrm{SO}_{4}$ solution under electrochemical potential control using EC-MCBJ. The conductance histogram exhibited series of peaks, suggesting the existence of the electronic shell effect at low conductance regime and atomic shell effect at high conductance regime. We could successfully observe shell effect and transition from the electronic to atomic shell effect under electrochemical potential control for the first time.

\section{Acknowledgement}

This work was partially supported by a Grant-in-Aid for Scientific Research from the Ministry of Education, Culture, Sports, Science and Technology, Japan.

\section{References}

[1] N. Agrait, A.L. Yeyati, and J.M. van Ruitenbeek, Physics Reports 377, 81-279 (2003).

[2] A.I. Mares and J.M. van Ruitenbeek, Phys. Rev. B 72, 205402 (2005).

[3] A.I. Mares, A.F. Otte, L.G. Soukiassian, R.H.M. Smit, and J.M. van Ruitenbeek, Phys. Rev. B 70, 073401 (2004).

[4] A.I. Yanson, I.K. Yanson, and J.M. van Ruitenbeek, Phys. Rev. Lett. 87, 216805 (2001).

[5] A.I. Yanson, I.K. Yanson, and J.M. van Ruitenbeek, Nature 400, 144-146 (1999).

[6] E. Medina, M. Diaz, N. León, C. Guerrero A. Hasmy, P.A. Serena, and J.L. Costa-Kramer, Phys. Rev. Lett, 91, 026802 (2003).

[7] I.K. Yanson, O.I. Shklyarevskii, Sz. Csonka, H. van Kempen, S. Speller, A.I. Yanson, and J.M. van Ruitenbeek, Phys. Rev. Lett. 95, 256806 (2005).

[8] M. Díaz, J.L. Costa-Kramer, E. Medina, A. Hasmy, and P. A Serena, Nanotechnology 14, 113-116 (2003).

[9] J. Li, T. Kanzaki, K. Murakoshi, and Y. Nakato, Appl. Phys. Lett. 81, 123-125 (2002).

[10] M. Kiguchi, T. Konishi, and K. Murakoshi, Appl. Phys. Lett. 87, 043104 (2005).

[11]M. Kiguchi and K. Murakoshi, Appl. Phys. Lett. 88, 253112 (2006).

[12] M. Kiguchi, T. Konishi, and K. Murakoshi, Phys. Rev. B 73, 125406 (2006).

[13]C.E. Bach, M. Giesen, and H. Ibach, Phys. Rev. Lett. 78, 4225 (1997).

[14] M. Kiguchi, N. Sekiguchi, and K. Murakoshi, in preparation.

[15]H. Uchida, M. Hiei, and M. Watanabe, $J$. Electroanal. Chem. 452, 97-106 (1998).

[16] E.A. Jagla and E. Tosatti, Phys. Rev. B 64, 205412 (2001).

[17] Y. Oshima and K. Mouri, H. Hirayama, and K. Takayanagi, J.Phys. Soc. Jpn. 75, 53705 (2006).

[18]H.X. He, C. Shu, C.Z. Li, and N.J. Tao, J. Electroanal. Chem. 522, 26-32 (2002). 